\title{
Análisis de los comportamientos parentales en relación a la estimulación motriz y el juego en niños de 1 a 4 años. Un estudio exploratorio
}

\section{Analysis of parental behaviors in relation to play and motor stimulation of their 1 to 4 years old children. An exploratory study}

LuCía PEIXOTO-PINO

Facultad de Ciencias de la Educación. Universidad de Santiago de Compostela. España Avda. Xoan XXIII, s/n (Campus Norte). 15782-Santiago de Compostela

lucia.peixoto@usc.es

\section{RAQUEL MARTÍNEZ-ROMÁN}

Grupo de Investigación REMOSS. Universidad de Vigo. España

raquelmartinezroman@hotmail.es

\section{JAVIER RICO-DÍAZ}

Facultad de Ciencias de la Educación. Universidad de Santiago de Compostela. España javier.rico.diaz@usc.es (Correspondencia en relación con este artículo, a este autor) ORCID: https://orcid.org/0000-0001-8858-145X

Recibido: 06-01-2019. Aceptado: 24-10-2019.

Cómo citar / Citation: Peixoto-Pino, L., Martínez-Román, R., y Rico-Díaz, J. (2019).

Análisis de los comportamientos parentales en relación a la estimulación motriz y el juego en niños de 1 a 4 años. Un estudio exploratorio, Ágora para la Educación Física y el Deporte, 21, 96-110.

DOI: https://doi.org/10.24197/aefd.0.2019.96-110

Resumen. Los comportamientos parentales juegan un importante papel en la adquisición de hábitos y costumbres en las primeras edades de los niños. En este trabajo hemos utilizado el Comprehensive Early Childhood Parenting Questionnaire (CECPAQ) -en concreto, los ítems del dominio de la estimulación- para explorar la influencia de los padres en la predisposición y costumbres hacia el juego y el movimiento por parte de sus hijos. Los resultados indican que, para la franja de 1 a 4 años, determinados comportamientos parentales tienden a estimular y favorecer el juego de los niños; que existen diferencias en relación al género (favorable a los niños) y en función del grado de urbanización.

Palabras clave. Estimulación; edad infantil; comportamientos parentales; desarrollo; juego. 


\begin{abstract}
Parental behaviors play a key role in the acquisition of habits and customs by their 1 to 4 years old children. This paper aims to explore children's habits towards play and movement. To do so, we've used the Comprehensive Early Childhood Parenting Questionnaire (CECPAQ), specifically the items related to the domain of stimulation. Data suggest that particular parental behaviors tend to stimulate and favor children's play during those early ages; however, some differences have been found in relation to gender (favorable to children) and to the (higher) level of urbanization.
\end{abstract}

Keywords: Stimulation; toddler and preschool child; parental behaviors; development; game.

\title{
INTRODUCCIÓN
}

Para que la población goce de un estado de salud adecuado, necesariamente debe adoptar conductas y comportamientos que favorezcan un estilo de vida saludable, y el entorno, particularmente la familia, es un elemento clave para favorecer la existencia de un contexto que facilite la adquisición de unos hábitos adecuados, ya desde la primera infancia (Ministerio de Sanidad, Servicios Sociales e Igualdad, 2014; Vaquero-Barba, Garay-Ibáñez-De-Elejalde y Rúiz-De-Arcaute-Graciano, 2015). La familia, el nivel socioeconómico del entorno, los espacios donde convivimos, los lugares disponibles para la práctica de actividad física, ejercicio físico y deporte, son elementos de gran relevancia para la determinación del estilo de vida de cada sujeto tanto en el presente como en el futuro.

A pesar de que las instituciones públicas, conscientes de esta situación, progresivamente avanzan hacia la promoción de la salud mediante la adopción de medidas preventivas que inciden en las conductas del individuo y de la sociedad en general sobre los determinantes de salud, como es la actividad físico-deportiva, el primer elemento clave en un estilo de vida activo es, desde que nacemos, la familia (World Health Organization, 2018).

Recientemente se ha publicado la Segunda Edición de una de las guías de referencia mundial sobre la práctica de actividad física, la "Physical Activity Guidelines for Americans, 2nd edition" (US Department of Health and Human Services, 2018), la cual pone de manifiesto la importancia de alentar a los niños en edad prescolar a moverse y a participar en juegos activos así como en actividades estructuradas. Como conclusión para este grupo de edad, señala que el 
objetivo razonable estaría en las tres horas por día de actividad de distintas intensidades, ligera, moderada y vigorosa. Indica también que, en este grupo de edad, los principales facilitadores de que dicho objetivo se consiga son la familia y los centros educativos -si el niño/a está escolarizado-. Se debe procurar fomentar experiencias físicamente activas en las que todos los participantes se sientan cómodos, disfruten de las actividades y en las que exista un componente educativo orientado a la adquisición del hábito (Vaquero-Barba, et al., 2015). Otro aspecto que se debe tener en cuenta es la necesidad de favorecer ya desde las primeras experiencias que los niños tomen la responsabilidad de su propio proceso de aprendizaje a la hora de afrontar actividades que supongan carga de actividad física (Wichmann, 2011).

La evaluación de los comportamientos parentales constituye un componente fundamental para la investigación del desarrollo de los niños (Verhoeven, Deković, Bodden, \& Van, 2017). Se han encontrado asociaciones entre el desarrollo de los niños cuando los padres proporcionan los medios para la estimulación de sus hijos. De hecho, los comportamientos y hábitos parentales pueden explicar entre el 20 y 50\% del desarrollo infantil (Zimmer-Gembeck \& Thomas, 2010). En relación al desarrollo, los niños en edad infantil que tienen una mayor estimulación motriz, alcanzan rangos más elevados en diversos aspectos del movimiento, como la coordinación y equilibrio (Costa, BarcalaFurelos, Abelairas-Gomez, y Arufe-Giraldez, 2015). En relación con la salud, es sabido el determinante papel que desempeñan en la obesidad infantil los comportamientos y actitudes sedentarias, y que son los países del sur de Europa (España y Grecia) los que presentan los índices de obesidad más altos, asociados principalmente a los hábitos de actividad física y al contexto socioeconómico (a mayor pobreza, mayor obesidad) (Van Stralen et al., 2012). Sin embargo, los índices de actividad física infantiles a nivel global parecen no estar asociados ni con el género ni con el estatus socioeconómico de los padres (De Craemer et al., 2012). El tiempo del que disponen los éstos también ha sido analizado, ya que la conciliación familiar es un condicionante clave de las actividades físicas que realizan los hijos (Fraguela-Vale, Lorenzo-Castiñeiras, \& VarelaGarrote, 2011). Aun así, no existen demasiadas evidencias sobre los comportamientos parentales influyentes en la estimulación motriz infantil y sobre los patrones de actividad física en el ámbito preescolar (De Craemer, McGregor, Androutsos, Manios, \& Cardon, 2018). 
El cuestionario para la comprensión de la crianza de los hijos, Comprehensive early childhood parenting questionnaire, fue validado para analizar los comportamientos de los padres que refuerzan la adaptación de los niños en una serie de dominios (soporte, estimulación, estructura, disciplina severa y disciplina positiva). El dominio de estimulación contiene una serie de ítems relacionados con conductas motrices y el juego. En nuestro ámbito de conocimiento no hay muchos estudios que focalicen su análisis en el ámbito psicomotor del cuestionario, ni en la franja de edad de 1 a 4 años, ni teniendo en cuenta el grado de urbanización de la residencia familiar. El objetivo de este trabajo es explorar la frecuencia de comportamientos parentales relacionados con la estimulación motriz en la etapa infantil.

\section{MATERIAL y Método}

Se realizó un estudio descriptivo de corte transversal, para conocer el comportamiento, actitudes lúdicas y motrices de un colectivo de progenitores con hijos comprendidos entre 1 y 4 años.

\subsection{Diseño del estudio}

Se empleó el cuestionario Comprehensive Early Childhood Parenting Questionnaire (CECPAQ) que ha sido validado para familias con hijos de entre 1 y 4 años (Verhoeven et al., 2017) con el fin de analizar la frecuencia de determinadas conductas ante la educación de los hijos.

El cuestionario tiene un total de 54 preguntas en las que se abordan constructos relacionados con la afectividad en las relaciones filiales, las interacciones sobre los juegos y los comportamientos parentales.

Un grupo de expertos conformado por profesores de universidad del ámbito de la educación, particularmente de Educación Física, maestros de Educación Infantil y maestros especialistas en Educación Física, discriminaron las preguntas orientadas a la estimulación motriz y al juego infantil; posteriormente, mediante un grupo de discusión se realizó la traducción al español. Al final de este proceso, se determinó un bloque de ítems conformado por 8 preguntas (Tabla I) 
Tabla I. Ítems del dominio “estimulación” del CECPAQ en su formato original y la traducción basada en la discusión de expertos

\begin{tabular}{|c|c|c|c|}
\hline $\begin{array}{l}\text { Orden del } \\
\text { ítem en el } \\
\text { CECPAQ }\end{array}$ & Pregunta original & $\begin{array}{l}\text { Codificación } \\
\text { del ítem para } \\
\text { este estudio }\end{array}$ & Traducción al español \\
\hline Q45 & $\begin{array}{l}\text { I encourage my child to play } \\
\text { alone and explore his/her } \\
\text { environment }\end{array}$ & P1 & $\begin{array}{l}\text { Animo a mi hijo/a a jugar solo } \\
\text { y explorar su entorno }\end{array}$ \\
\hline Q44 & $\begin{array}{l}\text { I take my child outside to play, } \\
\text { walk, or cycle }\end{array}$ & P2 & $\begin{array}{l}\text { Saco a mi hija/o afuera para } \\
\text { jugar, caminar o andar en } \\
\text { bicicleta. }\end{array}$ \\
\hline Q46 & $\begin{array}{l}\text { I regularly let my child play } \\
\text { with other children }\end{array}$ & P3 & $\begin{array}{l}\text { Habitualmente dejo que mi } \\
\text { hijo/a juegue con otros niños. }\end{array}$ \\
\hline Q47 & $\begin{array}{l}\text { I regularly let my child play } \\
\text { with adults, other than the } \\
\text { parents }\end{array}$ & $\mathbf{P 4}$ & $\begin{array}{l}\text { Habitualmente dejo que mi } \\
\text { hija/o juegue con adultos, } \\
\text { aparte de los padres. }\end{array}$ \\
\hline Q50 & $\begin{array}{l}\text { My child and I play together } \\
\text { with colorful toys made of } \\
\text { different materials (e.g., soft } \\
\text { toys, wooden blocks, rattle } \\
\text { book) }\end{array}$ & P5 & $\begin{array}{l}\text { Mi hijo/a y yo jugamos juntos } \\
\text { con juguetes variados hechos } \\
\text { de diferentes materiales (ej. } \\
\text { peluches, bloques de madera, } \\
\text { libros de sonidos, etc.) }\end{array}$ \\
\hline Q51 & $\begin{array}{l}\text { My child and I play together } \\
\text { with toys that can roll (e.g., } \\
\text { ball, cars, pull animal) }\end{array}$ & P6 & $\begin{array}{l}\text { Mi hija/o y yo jugamos con } \\
\text { juguetes que pueden rodar (ej. } \\
\text { pelotas, automóviles, animales } \\
\text { de tracción, etc.). }\end{array}$ \\
\hline Q52 & $\begin{array}{l}\text { My child and I play together } \\
\text { with building blocks, Lego, or } \\
\text { other stacking toys }\end{array}$ & P7 & $\begin{array}{l}\text { Mi hijo/a y yo jugamos con } \\
\text { bloques de construcción lego y } \\
\text { otros juguetes. }\end{array}$ \\
\hline Q54 & $\begin{array}{l}\text { My child and I play together } \\
\text { with musical toys (e.g., drum, } \\
\text { flute) }\end{array}$ & P8 & $\begin{array}{l}\text { Mi hijo/a y yo jugamos con } \\
\text { juguetes musicales (ej. tambor, } \\
\text { flauta, etc.) }\end{array}$ \\
\hline
\end{tabular}

\subsection{Participantes}

El cuestionario fue distribuido por email y redes sociales en un entorno controlado (comarca de Pontevedra), en un formato de respuesta online. El criterio de inclusión es que tuviesen un hijo en edad infantil, aunque para los efectos de este trabajo se contabilizó sólo aquellas respuestas con un niño de edad comprendida entre 1 y 4 años. Si tenían más de uno, se les solicitaba en el cuestionario que aclarasen la edad de aquél sobre el cual emitían sus respuestas. Este trabajo respetó los 
principios éticos de la Declaración de Helsinki. Los participantes fueron informados antes de realizar el formulario, siendo condición indispensable para continuar, la autorización del uso de los datos, de forma anónima y con únicos fines de investigación.

\subsection{Variables}

Las variables dependientes de este estudio fueron las derivadas de las respuestas de los ítems del cuestionario, presentadas en escala Likert de 6 niveles con las siguientes posibilidades: Nunca, Casi Nunca, A Veces, Frecuentemente, Casi Siempre, Siempre. Las variables independientes fueron el género y grado de urbanización de la residencia familiar, basado en el criterio del Instituto Gallego de Estadística (IGE, s.f.) para clasificar las poblaciones en función de su grado de urbanización, estableciéndose dos niveles: Grado de Urbanización Alto (GUA) y Grado de Urbanización Medio o Bajo (GMB).

\subsection{Análisis Estadístico}

El tratamiento de los datos se realizó con SPSS para Windows, versión 20 (SPSS Inc, IBM, EE.UU.). Se realizaron los siguientes análisis: a) Se analizó la normalidad de la muestra utilizando el test Kolmoronov-Smirnov; b) Se usó el Chi-cuadrado para la comparación de grupos y la V de Cramer para conocer el tamaño del efecto; c) las variables se describieron mediante frecuencias y porcentajes. Se estableció $\mathrm{p}<.05$ para todos los análisis.

\section{RESUltados}

Un total de 96 progenitores respondieron al cuestionario [80 madres (83.3\%), 16 padres (16.7\%)] refiriéndose a un total de 96 hijos [40 niñas (41.7\%), 56 niños (58.3\%)].

En la tabla II se muestran los resultados totales, así como desagregados por género. De forma genérica, en los ítems P1, P3 y P8 la opción mayoritaria de respuesta es siempre, sin embargo, en el análisis por género, esa tendencia no se cumple en P1 $\left(\chi^{2}=.005\right.$; VC $=$ Grande) [niñas 32,5\% vs niños 67.9\%], la diferencia en siempre es de $35.4 \%$ a favor de los niños, en cambio la opción más respondida para las niñas fue casi siempre. En la cuestión P8 ocurre lo mismo, pero sin significatividad 
estadística y tamaño del efecto medio $\left(\chi^{2}=.38\right.$; $\left.\mathrm{VC}=0.24\right)$, con una mayor tendencia al siempre en los varones como opción mayoritaria [niñas 22.5\% vs niños 39.3\%] y al casi siempre en las mujeres [niñas $30 \%$ vs niños 19.6\%]. En P2, P4, P5, P6 y P7, la opción de respuesta mayoritaria es casi siempre, sin embargo, esta tendencia cambia cuando se analiza por género. En las cuestiones P5, P6, P7 y P8 la respuesta preferida para los varones es siempre: P5 [24\% de diferencia niños vs niñas], P6 [16.4\% de diferencia niños vs niñas], P7 [13.9\% de diferencia niños vs niñas], P8 [16.8\% de diferencia niños vs niñas]. En 7 de las 8 cuestiones, los niños obtienen el mayor porcentaje en la opción siempre y las niñas tan sólo en una. Esta tendencia se puede apreciar en la Figura 1.

\title{
(Tabla II, página siguiente)
}

Tabla II. Respuestas relacionadas con el juego. Cuestionario CECPAQ. Totales y por género

\begin{abstract}
(P1: Animo a mi hijo/a a jugar solo y explorar su entorno. P2: Saco a mi hija/o afuera para jugar, caminar o andar en bicicleta. P3: Habitualmente dejo que mi hijo/a juegue con otros niños. P4: Habitualmente dejo que mi hija/o juegue con adultos, aparte de los padres. P5: Mi hijo/a y yo jugamos juntos con juguetes variados hechos de diferentes materiales (ej. peluches, bloques de madera, libros de sonidos etc.) P6: Mi hija/o y yo jugamos con juguetes que pueden rodar (ej. pelotas, automóviles, animales de tracción, etc.) P7: Mi hijo/a y yo jugamos con bloques de construcción lego y otros juguetes. P8: Mi hijo/a y yo jugamos con juguetes musicales (ej. tambor, flauta, etc.) TAMAÑO DEL (TE) EFECTO <0.06 Trivial / 0.06-0.18 Pequeño / 0.18-0.29 Medio / 0.29-1 Grande. CHI CUADRADO $\chi^{2}$ )
\end{abstract}




\begin{tabular}{|c|c|c|c|c|c|c|}
\hline & VARIABLE & $\begin{array}{l}\text { TODOS } \\
(n=96)\end{array}$ & $\begin{array}{l}\text { NIÑAS } \\
(n=40)\end{array}$ & $\begin{array}{l}\text { NIÑOS } \\
(n=56)\end{array}$ & $\chi^{2}$ & $\begin{array}{c}\text { V CRAMER } \\
\text { TE }\end{array}$ \\
\hline P1 & $\begin{array}{l}\text { Nunca } \\
\text { Casi Nunca } \\
\text { A Veces } \\
\text { Frecuentemente } \\
\text { Casi Siempre } \\
\text { Siempre }\end{array}$ & $\begin{array}{l}0(0 \%) \\
0(0 \%) \\
4(4.2 \%) \\
11(11.5 \%) \\
30(31.3 \%) \\
51(53.1 \%)\end{array}$ & $\begin{array}{l}0(0 \%) \\
0(0 \%) \\
2(5 \%) \\
8(20 \%) \\
17(42.5 \%) \\
13(32.5 \%)\end{array}$ & $\begin{array}{l}0(0 \%) \\
0(0 \%) \\
2(3.6 \%) \\
3(5.4 \%) \\
13(23.2 \%) \\
38(67.9 \%)\end{array}$ & .005 & 0.36 (Grande) \\
\hline P2 & $\begin{array}{l}\text { Nunca } \\
\text { Casi Nunca } \\
\text { A Veces } \\
\text { Frecuentemente } \\
\text { Casi Siempre } \\
\text { Siempre }\end{array}$ & $\begin{array}{l}0(0 \%) \\
0(0 \%) \\
3(3.1 \%) \\
19(19.8 \%) \\
52(54.2 \%) \\
22(22.9 \%) \\
\end{array}$ & $\begin{array}{l}0(0 \%) \\
0(0 \%) \\
1(2.5 \%) \\
9(22.5 \%) \\
25(62.5 \%) \\
5(12.5 \%) \\
\end{array}$ & $\begin{array}{l}0(0 \%) \\
0(0 \%) \\
2(3.6 \%) \\
10(17.9 \%) \\
27(48.2 \%) \\
17(30.4 \%) \\
\end{array}$ & .22 & 0.22 (Medio) \\
\hline P3 & $\begin{array}{l}\text { Nunca } \\
\text { Casi Nunca } \\
\text { A Veces } \\
\text { Frecuentemente } \\
\text { Casi Siempre } \\
\text { Siempre }\end{array}$ & $\begin{array}{l}0(0 \%) \\
1(1 \%) \\
3(3.1 \%) \\
8(8.3 \%) \\
20(20.8 \%) \\
64(66.7 \%)\end{array}$ & $\begin{array}{l}0(0 \%) \\
0(0 \%) \\
1(2.5 \%) \\
6(15 \%) \\
10(25 \%) \\
23(57.5 \%)\end{array}$ & $\begin{array}{l}0(0 \%) \\
1(1.8 \%) \\
2(3.6 \%) \\
2(3.6 \%) \\
10(17.9 \%) \\
41(73.2 \%)\end{array}$ & .22 & 0.25 (Medio) \\
\hline P4 & $\begin{array}{l}\text { Nunca } \\
\text { Casi Nunca } \\
\text { A Veces } \\
\text { Frecuentemente } \\
\text { Casi Siempre } \\
\text { Siempre }\end{array}$ & $\begin{array}{l}0(0 \%) \\
6(6.3 \%) \\
9(9.4 \%) \\
23(24 \%) \\
35(36.5 \%) \\
23(24 \%)\end{array}$ & $\begin{array}{l}0(0 \%) \\
2(5 \%) \\
4(10 \%) \\
9(22.5 \%) \\
16(40 \%) \\
9(22.5 \%)\end{array}$ & $\begin{array}{l}0(0 \%) \\
4(7.1 \%) \\
5(8.9 \%) \\
14(25.0 \%) \\
19(33.9 \%) \\
14(25.0 \%)\end{array}$ & .97 & 0.07 (Pequeño) \\
\hline P5 & $\begin{array}{l}\text { Nunca } \\
\text { Casi Nunca } \\
\text { A Veces } \\
\text { Frecuentemente } \\
\text { Casi Siempre } \\
\text { Siempre }\end{array}$ & $\begin{array}{l}0(0 \%) \\
1(1 \%) \\
1(1 \%) \\
13(13.5 \%) \\
42(43.8 \%) \\
39(40.6 \%)\end{array}$ & $\begin{array}{l}0(0 \%) \\
0(0 \%) \\
0(0 \%) \\
3(7.5 \%) \\
22(55 \%) \\
15(37.5 \%)\end{array}$ & $\begin{array}{l}0(0 \%) \\
1(1.8 \%) \\
1(1.8 \%) \\
10(17.9 \%) \\
20(35.7 \%) \\
24(61.5 \%)\end{array}$ & .25 & 0.24 (Medio) \\
\hline P6 & $\begin{array}{l}\text { Nunca } \\
\text { Casi Nunca } \\
\text { A Veces } \\
\text { Frecuentemente } \\
\text { Casi Siempre } \\
\text { Siempre }\end{array}$ & $\begin{array}{l}0(0 \%) \\
0(0 \%) \\
3(3.1 \%) \\
13(13.5 \%) \\
42(43.8 \%) \\
38(39.6 \%) \\
\end{array}$ & $\begin{array}{l}0(0 \%) \\
0(0 \%) \\
0(0 \%) \\
5(12.5 \%) \\
23(57.5 \%) \\
12(30 \%) \\
\end{array}$ & $\begin{array}{l}0(0 \%) \\
0(0 \%) \\
3(5.4 \%) \\
8(14.3 \%) \\
19(33.9 \%) \\
26(46.4 \%) \\
\end{array}$ & .08 & 0.08 (Pequeño) \\
\hline P7 & $\begin{array}{l}\text { Nunca } \\
\text { Casi Nunca } \\
\text { A Veces } \\
\text { Frecuentemente } \\
\text { Casi Siempre } \\
\text { Siempre }\end{array}$ & $\begin{array}{l}2(2.1 \%) \\
1(1 \%) \\
12(12.5 \%) \\
19(19.8 \%) \\
35(36.5 \%) \\
27(28.1 \%)\end{array}$ & $\begin{array}{l}2(5 \%) \\
0(0 \%) \\
7(17,5 \%) \\
6(15 \%) \\
17(42,5 \%) \\
8(20 \%)\end{array}$ & $\begin{array}{l}0(0 \%) \\
1(1.8 \%) \\
5(8.9 \%) \\
13(23.2 \%) \\
18(32.1 \%) \\
19(33.9 \%)\end{array}$ & .16 & 0.29 (Grande) \\
\hline P8 & $\begin{array}{l}\text { Nunca } \\
\text { Casi Nunca } \\
\text { A Veces } \\
\text { Frecuentemente } \\
\text { Casi Siempre } \\
\text { Siempre }\end{array}$ & $\begin{array}{l}1(1 \%) \\
5(5.2 \%) \\
18(18.8 \%) \\
18(18.8 \%) \\
23(24 \%) \\
31(32.3 \%) \\
\end{array}$ & $\begin{array}{l}0(0 \%) \\
2(5,0 \%) \\
10(25 \%) \\
7(17,5 \%) \\
12(30 \%) \\
9(22,5 \%) \\
\end{array}$ & $\begin{array}{l}1(1.8 \%) \\
3(5.4 \%) \\
8(14.3 \%) \\
11(19.6 \%) \\
11(19.6 \%) \\
22(39.3 \%)\end{array}$ & .38 & 0.24 (Medio) \\
\hline
\end{tabular}




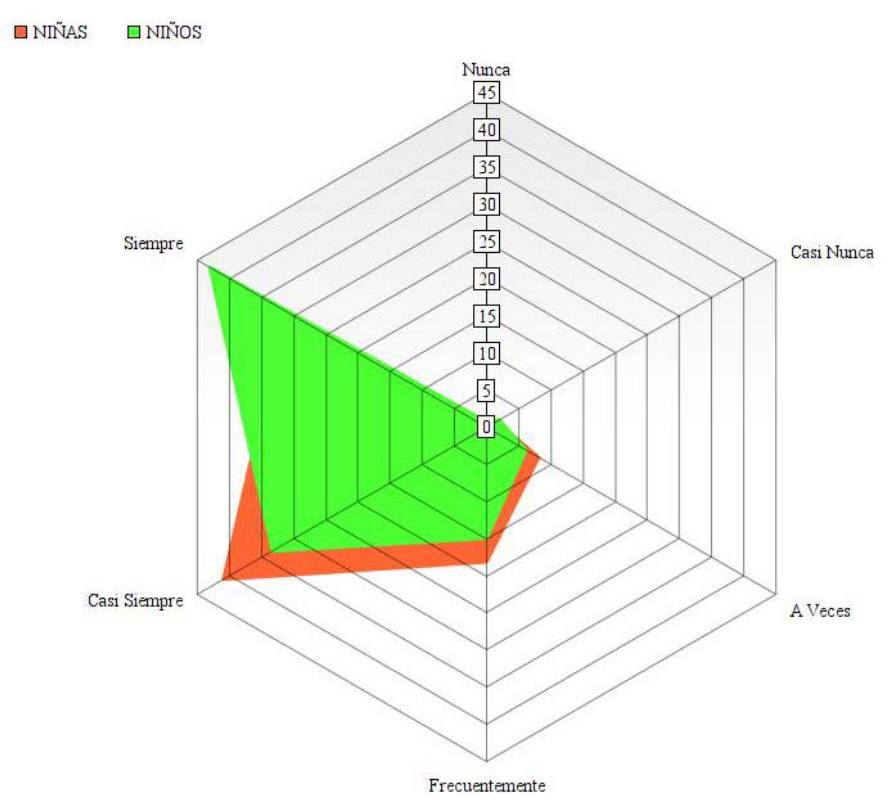

Figura 1. Gráfico de la tendencia de respuesta para el conjunto de ítems relacionados con el juego y actividad física infantil del CECPAQ desagregados por género.

En la tabla III se muestran los resultados desagregados por GU. Para el grupo GUA, en 6 de 8 ítems la respuesta mayoritaria es siempre. Se encuentra significatividad estadística en $\mathrm{P} 4$ con un tamaño del efecto grande $\left(\chi^{2}=.04 ; \mathrm{VC}=0.32\right)$. En este ítem para la categoría siempre, el grupo GUA obtiene un $17 \%$ más que GMB. Las cuestiones P1, P2, P3 y P7 coinciden en el porcentaje de respuesta mayoritaria (siempre o casi siempre), mientras que para las variables P4, P5, P6 y P8 que su máximo porcentaje fue la opción siempre para el grupo GUA obtienen una ventaja porcentual con respecto GMB de 17\%, 21\%, 13\% y 14.2\%. En la Figura 2 puede apreciarse esta tendencia.

(Tabla III, página siguiente)

Tabla III. Respuestas de los ítems relacionados con el juego el cuestionario CECPAQ, totales y desagregados por grado de urbanidad 


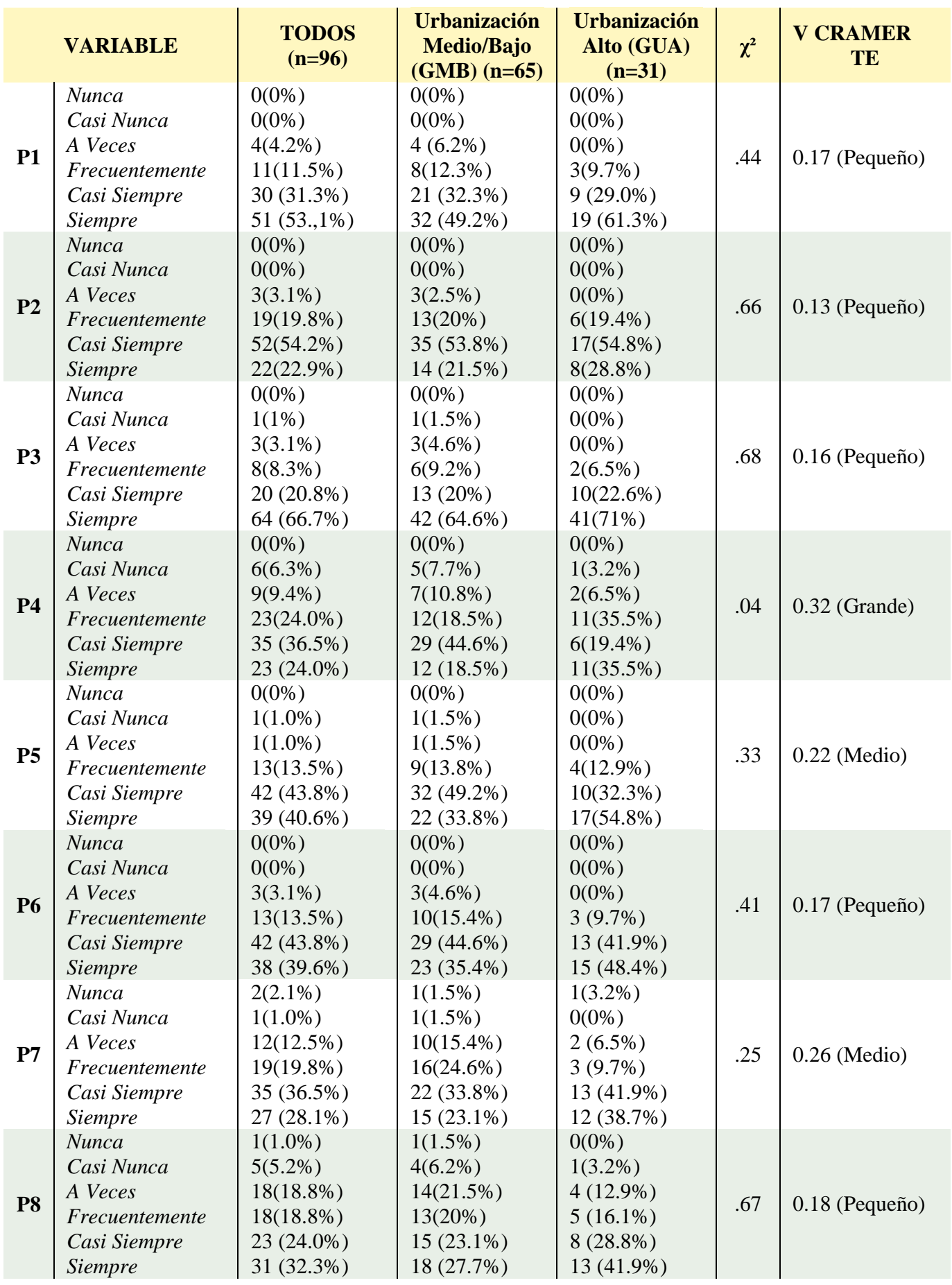


(P1: Animo a mi hijo/a a jugar solo y explorar su entorno. P2: Saco a mi hija/o afuera para jugar, caminar o andar en bicicleta. P3: Habitualmente dejo que mi hijo/a juegue con otros niños. P4: Habitualmente dejo que mi hija/o juegue con adultos, aparte de los padres. P5: Mi hijo/a y yo jugamos juntos con juguetes variados hechos de diferentes materiales (ej. peluches, bloques de madera, libros de sonidos etc.) P6: Mi hija/o y yo jugamos con juguetes que pueden rodar (ej. pelotas, automóviles, animales de tracción, etc.) P7: Mi hijo/a y yo jugamos con bloques de construcción lego y otros juguetes. P8: Mi hijo/a y yo jugamos con juguetes musicales (ej. tambor, flauta, etc.) TAMAÑO DEL (TE) EFECTO <0.06 Trivial / 0.06-0.18 Pequeño / 0.18-0.29 Medio / 0.29-1 Grande. CHI CUADRADO $\chi^{2}$ )

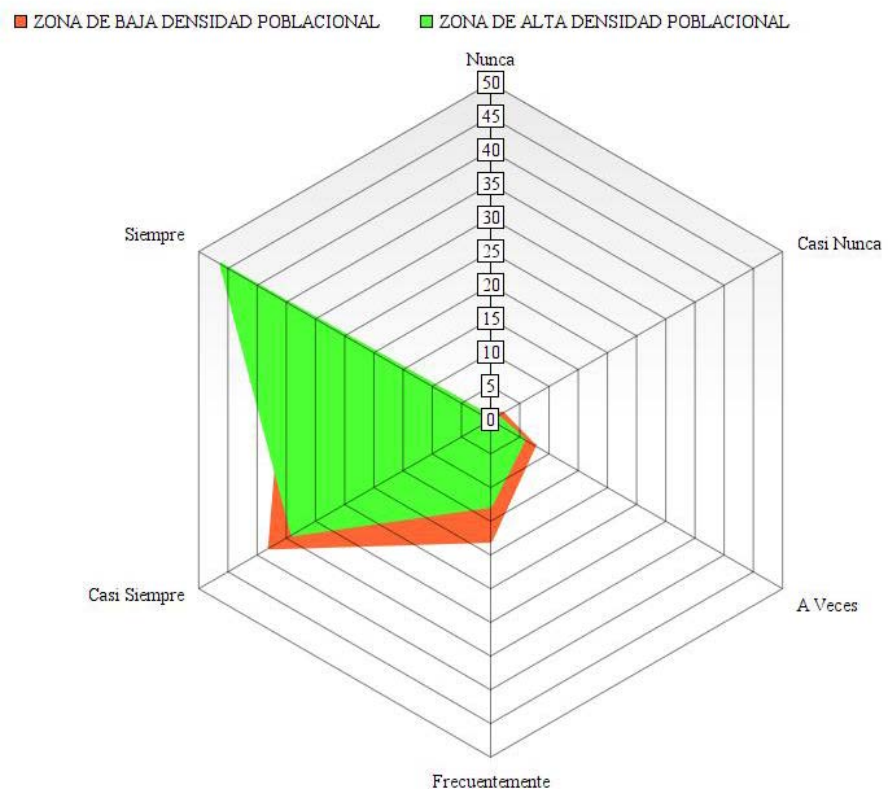

Figura 2. Gráfico de la tendencia de respuesta para el conjunto de ítems relacionados con el juego y actividad física infantil del CECPAQ desagregados por grado de urbanidad

\section{DisCUSIÓN Y CONCLUSIONES}

El objetivo de este estudio fue analizar los comportamientos parentales de aquellos ítems del CECPAQ que están relacionados con el juego, dentro del dominio de la estimulación. 
La estimulación motriz (Costa et al., 2015) y los comportamientos que los padres tienen con los hijos en relación a la actividad física (Zimmer-Gembeck \& Thomas, 2010) tienen un efecto positivo sobre su desarrollo. En este sentido, la mayoría de las familias han optado por responder la opción de siempre o casi siempre. La suma de los porcentajes de estas dos opciones supera en todos los casos el $50 \%$ de las respuestas y alcanza en algunos casos el 80\%. Los resultados sugieren que los padres de este estudio no son sobreprotectores (el $87 \%$ deja que jueguen con otros niños siempre o casi siempre fuera del ámbito escolar). Llama la atención que no se obtenga el mayor número de respuestas cuando se trata de sacar a los niños fuera del hogar para jugar, caminar o andar en bicicleta. Este resultado puede tener numerosas explicaciones, especialmente relacionadas con la meteorología, por tratarse de una comarca especialmente lluviosa. Un reciente estudio multicéntrico encontró una relación entre el clima y la actividad física, esto es, a peores condiciones, menos actividad (Harrison et al., 2017). Otra explicación razonable a este hallazgo tiene que ver con el tiempo del que disponen los padres y su posibilidad de conciliación, o con el afianzamiento del sedentarismo a nivel familiar, o el cambio del comportamiento en el ocio (Fraguela-Vale et al., 2011).

Otro aspecto relevante es el género. Para la cohorte de 1 a 4 años no se han encontrado diferencias significativas entre niños y niñas importantes (sólo en un ítem), sin embargo, en los varones, el mayor rango de respuesta "siempre" ha estado más veces presente y se ha encontrado una mayor asociación de las niñas pequeñas de esta edad con el sedentarismo (Carson \& Kuzik, 2017).

Sobre la actividad física infantil, un factor que está siendo analizado con especial énfasis en los últimos años es el lugar de residencia, tanto por sus implicaciones socioeconómicas como por los espacios y oferta de ocio activo (De Craemer et al., 2012; Van Stralen et al., 2012). Ante la idea generalizada que el pueblo o la aldea, por sus características más naturales, puede ser generador de un mayor índice de actividad, se ha encontrado resultados contradictorios, es decir asociaciones positivas con un mayor grado de urbanidad o densidad poblacional, posiblemente relacionado también a una amplia oferta de ocio o vida social (Rodríguez-Romo, Garrido-Muñoz, Lucía, Mayorga, \& Ruiz, 2013). Cuando se trata de jugar con otros adultos, llama la atención que la respuesta siempre es opción preferente en niños correspondientes a GUA, 
lo que hace pensar que quizá un entorno más social ofrece más confianza para dejar a los niños a cargo de otras personas (otros padres, vecinos).

Este estudio tiene limitaciones que deben ser señaladas. El análisis se circunscribe a una comarca en concreto, pudiendo no ser extrapolable estos resultados a otras poblaciones. El tamaño muestral no es representativo del universo, si bien no hay estudios previos que se hayan centrado en la estimulación de los ítems del CECPAQ. El cuestionario está validado en lengua inglesa, en español se ha propuesto una traducción, siendo el producto final 8 ítems basados en el consenso de expertos. En el futuro, sería interesante realizar un análisis de fiabilidad y validez de la traducción.

Por lo tanto, a la vista de los datos, parece que existe una tendencia generalizada por parte de las familias a potenciar los comportamientos y hábitos motrices en los niños de 1 a 4 años, ligeramente superior para los niños, especialmente en el juego de elementos que ruedan (tipo coches o pelotas) y en zonas con un grado de urbanización alto. Son necesarios más estudios para conocer el tiempo y calidad de esta estimulación y sus efectos a largo plazo, tanto en el desarrollo como en la salud.

\section{BIBLIOGRAFÍA}

Carson, V., \& Kuzik, N. (2017). Demographic correlates of screen time and objectively measured sedentary time and physical activity among toddlers: a cross-sectional study. BMC Public Health, 17(1), 187. Recuperado en https://bmcpublichealth.biomedcentral.com/track/pdf/10.1186/s12889-017$\underline{4125-y}$ el 16 de diciembre de 2018. doi: 10.1186/s12889-017-4125-y

Costa, H. J. T., Barcala-Furelos, R., Abelairas-Gomez, C., \& Arufe-Giraldez, V. (2015). The influence of a structured physical education plan on preschool children's psychomotor development profiles. Australasian Journal of Early Childhood, 40(2), 68-77. doi: 10.1177/183693911504000209

De Craemer, M., De Decker, E., De Bourdeaudhuij, I., Vereecken, C., Deforche, B., Manios, Y., ... ToyBox-study group. (2012). Correlates of energy balance-related behaviours in preschool children: a systematic review. Obesity Reviews: An Official Journal of the International Association for the Study of Obesity, 13 Suppl 1, 13-28. Recuperado el 2 de diciembre de 2018 en https://doi.org/10.1111/j.1467-789X.2011.00941.x 
De Craemer, M, McGregor, D., Androutsos, O., Manios, Y., \& Cardon, G. (2018). Compliance with 24-h Movement Behaviour Guidelines among Belgian Pre-School Children: The ToyBox-Study. International Journal of Environmental Research and Public Health, 15(10). Recuperado el 2 de diciembre de 2018 en https://doi.org/10.3390/ijerph15102171

Fraguela-Vale, R., Lorenzo-Castiñeiras, J., \& Varela-Garrote, L. (2011). Conciliación y actividad física de ocio en familias con hijos en Educación Primaria. Implicaciones para la infancia. Revista de investigación en educación, 9(2), 162-173.

Harrison, F., Goodman, A., van Sluijs, E. M. F., Andersen, L. B., Cardon, G., Davey, R., ... on behalf the ICAD collaborators. (2017). Weather and children's physical activity; how and why do relationships vary between countries? The International Journal of Behavioral Nutrition and Physical Activity, 14(1), 74. Recuperado el 15 de noviembre de 2018 en https://doi.org/10.1186/s12966-017-0526-7

IGE - Principais resultados. (s.f.). Recuperado el 9 de diciembre de 2018, de http://www.ige.eu/igebdt/esqv.jsp?ruta=verPpalesResultados.jsp?OP $=1 \& \mathrm{~B}$ $=1 \& \mathrm{M}=\& \mathrm{COD}=8053 \& \mathrm{R}=0[$ all $] \& \mathrm{C}=1[$ all $] \& \mathrm{~F}=\& \mathrm{~S}=\& \mathrm{SCF}=$

Ministerio de Sanidad, Servicios Sociales e Igualdad. (2014). Actividad Física y Salud de 3 a 6 años Guía para docentes de Educación Infantil. Recuperado el 2 de diciembre de 2018 en

http://www.mscbs.gob.es/ca/profesionales/saludPublica/prevPromocion/Es trategia/docs/GuiaAF_3_6anos_docentes.pdf

Rodríguez-Romo, G., Garrido-Muñoz, M., Lucía, A., Mayorga, J. I., \& Ruiz, J. R. (2013). Asociación entre las características del entorno de residencia y la actividad física. Gaceta Sanitaria, 27(6), 487-493. Recuperado el 10 octubre de 2018 en: https://doi.org/10.1016/j.gaceta.2013.01.006

US Department of Health and Human Services (2018). Physical Activity Guidelines for Americans 2nd edition. Recuperado el 2, diciembre, 2018 https://health.gov/paguidelines/secondedition/pdf/Physical Activity Guidelines 2nd edition.pdf

Van Stralen, M. M., te Velde, S. J., van Nassau, F., Brug, J., Grammatikaki, E., Maes, L., ... ToyBox-study group. (2012). Weight status of European preschool children and associations with family demographics and energy 
balance-related behaviours: a pooled analysis of six European studies. Obesity Reviews: An Official Journal of the International Association for the Study of Obesity, 13 Suppl 1, 29-41. Recuperado el 15 de noviembre de 2018 en https://doi.org/10.1111/j.1467-789X.2011.00959.x

Vaquero-Barba, A., Garay-Ibáñez-De-Elejalde, B., y Rúiz-De-ArcauteGraciano, J. (2015). La importancia de las experiencias positivas y placenteras en la promoción de la actividad física orientada hacia la salud. Ágora para la Educación Física y el Deporte, 17(2), 168-181. Recuperado 7, mayo, 2018: https://dialnet.unirioja.es/servlet/articulo?codigo=5180353

Verhoeven, M., Deković, M., Bodden, D., \& Van Bar, A. (2017). Development and initial validation of the comprehensive early childhood parenting questionnaire (CECPAQ) for parents of 1-4 year-olds. European Journal of Developmental Psychology, 14(2), 233-247. Recuperado el 2 de diciembre de 2018 en https://doi.org/10.1080/17405629.2016.1182017

Wichmann, K. (2011). La adquisición de habilidades motrices. Ágora para la Educación Física y el Deporte, 13(1), 87-110. Recuperado el 2 de octubre de 2018 en https://dialnet.unirioja.es/servlet/articulo?codigo=3449536

World Health Organization. (2018). Global action plan on physical activity 2018-2030: more active people for a healthier world. Geneva. Recuperado http://apps.who.int/iris/bitstream/handle/10665/272722/9789241514187eng.pdf el 16 de diciembre de 2018

Zimmer-Gembeck, M. J., \& Thomas, R. (2010). Parents, parenting and toddler adaptation: Evidence from a national longitudinal study of Australian children. Infant Behavior and Development, 33(4), 518-529. Recuperado el 16 de diciembre de 2018 en https://doi.org/10.1016/j.infbeh.2010.07.004 